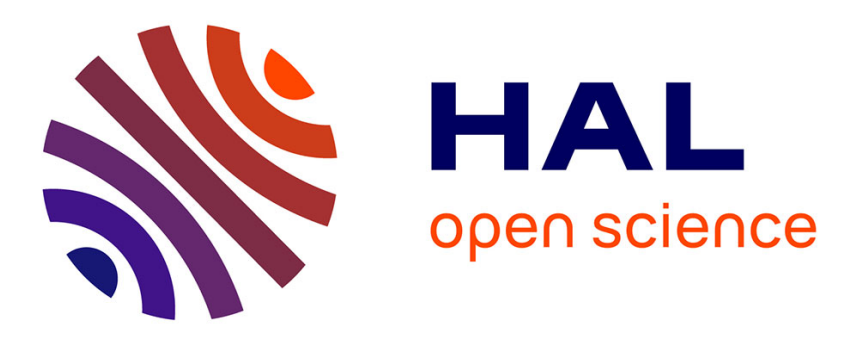

\title{
Max-min SINR low complexity transceiver design for single cell massive MIMO
}

\author{
Houssem Sifaou, Abla Kammoun, Luca Sanguinetti, Merouane Debbah, \\ Mohamed-Slim Alouini
}

\section{To cite this version:}

Houssem Sifaou, Abla Kammoun, Luca Sanguinetti, Merouane Debbah, Mohamed-Slim Alouini. Maxmin SINR low complexity transceiver design for single cell massive MIMO. 17th International Workshop on Signal Processing Advances in Wireless Communications (SPAWC), Jul 2016, Edinburgh, United Kingdom. 10.1109/SPAWC.2016.7536729 . hal-01789306

\section{HAL Id: hal-01789306 \\ https://hal-centralesupelec.archives-ouvertes.fr/hal-01789306}

Submitted on 25 Jul 2018

HAL is a multi-disciplinary open access archive for the deposit and dissemination of scientific research documents, whether they are published or not. The documents may come from teaching and research institutions in France or abroad, or from public or private research centers.
L'archive ouverte pluridisciplinaire HAL, est destinée au dépôt et à la diffusion de documents scientifiques de niveau recherche, publiés ou non, émanant des établissements d'enseignement et de recherche français ou étrangers, des laboratoires publics ou privés. 


\title{
Max-Min SINR Low Complexity Transceiver Design for Single Cell Massive MIMO
}

\author{
Houssem Sifaou*, Abla Kammoun*, Luca Sanguinetti ${ }^{\dagger \ddagger}$, Mérouane Debbah ${ }^{\ddagger \S}$, Mohamed-Slim Alouini* \\ *Electrical Engineering Department, King Abdullah University of Science and Technology, Thuwal, Saudi Arabia. \\ †Dipartimento di Ingegneria dell'Informazione, University of Pisa, Pisa, Italy. \\ ${ }_{\ddagger}^{\ddagger}$ Large Networks and System Group (LANEAS), CentraleSupélec, Université Paris-Saclay, Gif-sur-Yvette, France.

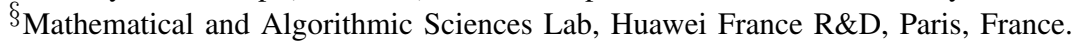

\begin{abstract}
This work focuses on large scale multi-user MIMO systems in which the base station (BS) outfitted with $M$ antennas communicates with $K$ single antenna user equipments (UEs). In particular, we aim at designing the linear precoder and receiver that maximizes the minimum signal-to-interference-plus-noise ratio (SINR) subject to a given power constraint. To gain insights into the structure of the optimal precoder and receiver as well as to reduce the computational complexity for their implementation, we analyze the asymptotic regime where $M$ and $K$ grow large with a given ratio and make use of random matrix theory (RMT) tools to compute accurate approximations. Although simpler, the implementation of the asymptotic precoder and receiver requires fast inversions of large matrices in every coherence period. To overcome this issue, we apply the truncated polynomial expansion (TPE) technique to the precoding and receiving vector of each UE and make use of RMT to determine the optimal weighting coefficients on a per-UE basis that asymptotically solve the maxmin SINR problem. Numerical results are used to show that the proposed TPE-based precoder and receiver almost achieve the same performance as the optimal ones while requiring a lower complexity.
\end{abstract}

Index Terms-Massive MIMO systems, linear transceivers, low complexity, truncated polynomial expansion, random matrix theory.

\section{INTRODUCTION}

Large-scale multiple-input multiple-output (MIMO) systems, better known as massive MIMO systems, are considered as a promising technique for next generation cellular networks [1]-[4]. The massive MIMO technology aims at evolving the conventional base stations (BSs) by using arrays with a hundred or more small dipole antennas. This allows for coherent multi-user MIMO transmission where tens of users can be multiplexed in both the uplink (UL) and downlink (DL) of each cell. The problem of designing precoder and receiver techniques for massive MIMO systems is receiving a lot of attention. Among the different optimization criteria, we distinguish the transmit power minimization [5]-[8] and the maximization of the minimum signal-to-interferenceplus-noise ratio (SINR) [9], [10]. The latter is the focus of this work. In particular, we consider a single-cell large-scale MIMO system in which the BS makes use of $M$ antennas to communicate with $K$ single-antenna user equipments (UEs).

L. Sanguinetti and M. Debbah were supported by the ERC Starting Grant 305123 MORE. This research was also partially supported by the research project 5GIOTTO funded by the University of Pisa.
Under the assumption of perfect channel state information (CSI) at the BS, in [9] it is shown that the optimal linear precoder (OLP) for the max-min SINR problem is closely related to the optimal linear receiver (OLR) as it can be computed exploiting the UL-DL duality principle. The latter allows to convert the DL optimization problem into its equivalent counterpart in the dual UL variables. The OLP is then found in the form of a fixed-point problem whose solution corresponds to the powers allocated to the UEs in the dual UL network. Although numerically feasible, the above approach does not provide any insight into the structure of both OLP and OLR. Moreover, their implementation requires the computation of the inverse of a matrix for any new realization of propagation channels, which is too computationally cumbersome when the network size becomes large (as envisioned in 5G networks).

To overcome this issue, we follow the same approach of other recent works [11], [12] and consider the asymptotic regime in which $M$ and $K$ grow large with a given ratio. This allows to provide accurate approximations of the parameters involved in the implementation of OLP and OLR, which turn out to depend only on the long-term channel attenuations of the UEs. This provides some insights on how these parameters affect the design of OLP and OLR but does not solve the issue concerning the computation of the matrix inverse. To tackle this problem, we resort to the truncated polynomial expansion (TPE) technique, which has been recently applied to reduce the complexity of the RZF precoder in [6], [13] and the MMSE filter in [14], [15]. However, in all these works the TPE concept is applied using the same weighting coefficients for all UEs. This limits the number of degrees of freedom with an ensuing degradation of the system performance. In light of this observation, we apply the TPE on a per-UE basis. More specifically, this amounts to applying the TPE concept to each vector of the precoding and receiving matrices rather than to the whole matrices themselves. This leads to the socalled user specific TPE (US-TPE) receiver and precoder for which approximations of the resulting SINRs are computed through the asymptotic analysis. These results are then used to optimize the US-TPE parameters in order to maximize the minimum SINR over all UEs in the DL and UL. Interestingly, the optimization problem can be cast as the max-min problems previously studied in [7], [9], [10]. Its solution leads to a novel US-TPE precoder and receiver, which are shown by 
simulations to achieve the same performances as OLP and OLR.

\section{SYSTEM MODEL}

We consider a single-cell massive MIMO system in which the BS equipped with $M$ antennas communicates with $K$ single antenna UEs, randomly selected from a large set of UEs within the coverage area. It is assumed in massive MIMO that $M>K$. We denote by $\mathbf{h}_{k} \in \mathbb{C}^{N}$ the channel vector of UE $k$ and assume that

$$
\mathbf{h}_{k}=\sqrt{\beta_{k}} \mathbf{z}_{k}
$$

where $\mathbf{z}_{k} \in \mathbb{C}^{N}$ is the small-scale fading channel assumed to be Gaussian with zero mean and unit covariance and $\beta_{k}$ accounts for the corresponding large-scale channel fading or path loss. The matrix collecting all UEs channel vectors will be denoted by $\mathbf{H}=\left[\mathbf{h}_{1}, \cdots, \mathbf{h}_{K}\right]$.

\section{A. Downlink}

Denoting by $\mathbf{g}_{i} \in \mathbb{C}^{N}$ the precoding vector associated to $\mathrm{UE} i$, the signal received at UE $k$ can be written as

$$
y_{k}=\sqrt{\rho} \mathbf{h}_{k}^{H} \mathbf{g}_{k} s_{k}+\sum_{i=1, i \neq k}^{K} \sqrt{\rho} \mathbf{h}_{k}^{H} \mathbf{g}_{i} s_{i}+n_{k}
$$

where $s_{i}$ is the signal intended to user $i$, assumed independent across $i$, with zero-mean and unit variance, $n_{k} \sim \mathcal{C N}(0,1)$ accounts for the additive Gaussian noise, and $\rho$ is the effective signal-to-noise ratio (SNR). The SINR at UE $k$ is thus given by:

$$
\operatorname{SINR}_{k}^{\mathrm{dl}}=\frac{\rho\left|\mathbf{h}_{k}^{H} \mathbf{g}_{k}\right|^{2}}{\sum_{i=1, i \neq k}^{K} \rho\left|\mathbf{h}_{k}^{H} \mathbf{g}_{i}\right|^{2}+1}
$$

and the average transmit power per UE is:

$$
P=\frac{1}{K} \operatorname{tr}\left(\mathbf{G G}^{H}\right)
$$

where $\mathbf{G}=\left[\mathbf{g}_{1}, \ldots, \mathbf{g}_{K}\right]$ denotes the precoding matrix. The latter is chosen as the solution of the following max-min SINR problem:

$$
\max _{\mathbf{g}_{1}, \ldots, \mathbf{g}_{K}} \min _{k} \frac{\mathrm{SINR}_{k}^{\mathrm{dl}}}{\gamma_{k}} \quad \text { s.t. } \quad \frac{1}{K} \operatorname{tr}\left(\mathbf{G G} \mathbf{G}^{H}\right) \leq P_{\max }
$$

where $\gamma_{k}$ is a factor reflecting the priority assigned to UE $k$ and $P_{\max }$ is the power constraint at the BS. In [9], the solution $\mathbf{g}_{k}^{\star}$ of the above problem is found to be $\mathbf{g}_{k}^{\star}=\sqrt{p_{k}^{\star} / K} \mathbf{u}_{k}^{\star}$ with

$$
\mathbf{u}_{k}^{\star}=\frac{\left(\frac{\rho}{K} \sum_{i \neq k} q_{i}^{\star} \mathbf{h}_{i} \mathbf{h}_{i}^{H}+\mathbf{I}_{M}\right)^{-1} \mathbf{h}_{k}}{\left\|\left(\frac{\rho}{K} \sum_{i \neq k} q_{i}^{\star} \mathbf{h}_{i} \mathbf{h}_{i}^{H}+\mathbf{I}_{M}\right)^{-1} \mathbf{h}_{k}\right\|}
$$

where the scalars $\left\{q_{\ell}^{\star}\right\}$ are obtained as the unique positive solution to the following fixed-point equations:

$$
q_{\ell}^{\star}=\frac{\gamma_{\ell} K P_{\max }}{\sum_{n=1}^{K} \frac{\gamma_{n} \mathbf{h}_{\ell}^{H}\left(\frac{\rho}{K} \sum_{k \neq \ell} q_{k}^{\star} \mathbf{h}_{k} \mathbf{h}_{k}^{H}+\mathbf{I}_{M}\right)^{-1} \mathbf{h}_{\ell}}{\mathbf{h}_{n}^{H}\left(\frac{\rho}{K} \sum_{k \neq n} q_{k}^{\star} \mathbf{h}_{k} \mathbf{h}_{k}^{H}+\mathbf{I}_{M}\right)^{-1} \mathbf{h}_{n}}}
$$

The optimal powers $\left\{p_{k}^{\star}\right\}$ are such that

$$
\frac{\operatorname{SINR}_{1}^{\mathrm{dl}}}{\gamma_{1}}=\cdots=\frac{\operatorname{SINR}_{K}^{\mathrm{dl}}}{\gamma_{K}} .
$$

Letting $\tau^{\star}=\frac{\mathrm{SINR}_{1}^{\mathrm{dl}}}{\gamma_{1}}$, it turns out that $\mathbf{p}^{\star}=\left[p_{1}^{\star}, \cdots, p_{K}^{\star}\right]$ is given by:

$$
\mathbf{p}^{\star}=\frac{\tau^{\star}}{\rho}\left(\mathbf{I}_{K}-\tau^{\star} \boldsymbol{\Gamma} \mathbf{F}\right)^{-1} \boldsymbol{\Gamma} \mathbf{1}_{K}
$$

where $\boldsymbol{\Gamma}=\operatorname{diag}\left(\frac{K \gamma_{1}}{\left|\mathbf{h}_{1}^{H} \mathbf{g}_{1}\right|^{2}}, \cdots, \frac{K \gamma_{K}}{\left|\mathbf{h}_{K}^{H} \mathbf{g}_{K}\right|^{2}}\right)$ and $\mathbf{F} \in \mathbb{C}^{K \times K}$ has entries given by

$$
[\mathbf{F}]_{k, i}=\left\{\begin{array}{lll}
0 & \text { if } & k=i \\
\frac{1}{K}\left|\mathbf{h}_{k}^{H} \mathbf{u}_{i}\right|^{2} & \text { if } & k \neq i
\end{array}\right.
$$

\section{B. Uplink}

From the uplink-downlink duality shown in [9], it is known that the vectors $\left\{\mathbf{u}_{k}^{\star}\right\}$ and scalars $\left\{q_{k}^{\star}\right\}$ may be thought as the solution of the following uplink max-min SINR problem:

$$
\max _{\substack{\mathbf{u}_{1}, \ldots, \mathbf{u}_{K} \\ q_{1}, \ldots, q_{K}}} \min _{k} \frac{\mathrm{SINR}_{k}^{\mathrm{ul}}}{\gamma_{k}} \quad \text { s.t. } \quad \frac{1}{K} \sum_{k=1}^{K} q_{k} \leq P_{\max }
$$

with $\mathrm{SINR}_{k}^{\mathrm{ul}}$ being given by

$$
\operatorname{SINR}_{k}^{\mathrm{ul}}=\frac{\rho \frac{q_{k}}{K}\left|\mathbf{u}_{k}^{H} \mathbf{h}_{k}\right|^{2}}{\sum_{i=1, i \neq k}^{K} \rho \frac{q_{i}}{K}\left|\mathbf{u}_{k}^{H} \mathbf{h}_{i}\right|^{2}+\left\|\mathbf{u}_{k}\right\|^{2}} .
$$

From (6), it easily follows that the vector $\mathbf{u}_{k}$ coincides with the minimum-mean-square-error (MMSE) receiver that maximizes $\mathrm{SINR}_{k}^{\mathrm{ul}}$.

\section{LARGE SYSTEM ANALYSIS}

As shown above, the optimal linear precoder (OLP) and the optimal linear receiver (OLR) are parametrized by the scalars $\left\{q_{k}^{\star}\right\}$ and $\left\{p_{k}^{\star}\right\}$ where $\left\{q_{k}^{\star}\right\}$ need to be evaluated by an iterative procedure due to the fixed-point equations in (7). This is a computationally demanding task when $M$ and $K$ are large since the matrix inversion operation in (7) must be recomputed at every iteration. Moreover, computing $\left\{q_{k}^{\star}\right\}$ as the fixed point of (7) does not provide any insights into the optimal structure of both $\left\{q_{k}^{\star}\right\}$ and $\left\{p_{k}^{\star}\right\}$. In addition, the parameter values depend directly on the channel vectors $\left\{\mathbf{h}_{k}\right\}$ and change at the same pace as the small-scale fading (i.e., at the order of milliseconds). To overcome these issues, we exploit the statistical distribution of $\left\{\mathbf{h}_{k}\right\}$ and the large values of $M, K$ to compute deterministic approximations of $\left\{q_{k}^{\star}\right\}$ and $\left\{p_{k}^{\star}\right\}$. A major result of this work is as follows:

Theorem 1. Let $\bar{\tau}$ be the unique positive solution to the following fixed point equation:

$$
\bar{\tau}=\frac{P_{\max } \rho\left[\frac{M}{K}-\frac{1}{K} \sum_{i=1}^{K} \frac{\gamma_{i} \bar{\tau}}{1+\gamma_{i} \bar{\tau}}\right]}{\frac{1}{K} \sum_{i=1}^{K} \frac{\gamma_{i}}{\beta_{i}}} .
$$

If $M$ and $K$ grow large with the same pace, then

$$
\tau^{\star}-\bar{\tau} \stackrel{\text { a.s. }}{\longrightarrow} 0
$$


and

$$
\begin{aligned}
& \max _{k}\left|q_{k}^{*}-\bar{q}_{k}\right| \stackrel{\text { a.s. }}{\longrightarrow} 0 \\
& \max _{k}\left|p_{k}^{*}-\bar{p}_{k}\right| \stackrel{\text { a.s. }}{\longrightarrow} 0
\end{aligned}
$$

where $\bar{q}_{k}$ and $\bar{p}_{k}$ are given by

$$
\begin{aligned}
\bar{q}_{k} & =\frac{\gamma_{k} \bar{\tau}}{\rho \beta_{k}\left(\frac{M}{K}-\frac{1}{K} \sum_{i=1}^{K} \frac{\gamma_{i} \bar{\tau}}{1+\gamma_{i} \bar{\tau}}\right)} \\
\bar{p}_{k} & =\frac{\gamma_{k} \bar{\tau}\left(\frac{\rho \beta_{k} P_{\max }}{\left(1+\gamma_{k} \bar{\tau}\right)^{2}}+1\right)}{\rho \beta_{k}\left(\frac{M}{K}-\frac{1}{K} \sum_{i=1}^{K} \frac{\left(\gamma_{i} \bar{\tau}\right)^{2}}{\left(1+\gamma_{i} \bar{\tau}\right)^{2}}\right)} .
\end{aligned}
$$

Proof: The proof is omitted due to space limitations but can be found in [16].

Theorem 1 states that the performance remains the same if $\left\{q_{k}^{*}\right\}$ and $\left\{p_{k}^{*}\right\}$ are replaced by $\left\{\bar{q}_{k}\right\}$ and $\left\{\bar{p}_{k}\right\}$. In doing so, we obtain the so-called asymptotically OLP (A-OLP) for which $\overline{\mathbf{g}}_{k}=\sqrt{\bar{p}_{k} / K} \overline{\mathbf{u}}_{k}$ and

$$
\overline{\mathbf{u}}_{k}=\frac{\left(\frac{\rho}{K} \sum_{i \neq k} \bar{q}_{i} \mathbf{h}_{i} \mathbf{h}_{i}^{H}+\mathbf{I}_{M}\right)^{-1} \mathbf{h}_{k}}{\left\|\left(\frac{\rho}{K} \sum_{i \neq k} \bar{q}_{i} \mathbf{h}_{i} \mathbf{h}_{i}^{H}+\mathbf{I}_{M}\right)^{-1} \mathbf{h}_{k}\right\|} .
$$

The asymptotically OLR (A-OLR) easily follows. From a practical standpoint, the use of $\left\{\bar{q}_{k}\right\}$ and $\left\{\bar{p}_{k}\right\}$ largely simplifies the implementation of A-OLP and A-OLR as their computation requires only the knowledge of the large scale channel statistics and must be performed only once per coherence period (rather than at the same pace as the small-scale fading). Despite being simplified, the implementation of AOLP and A-OLR still requires the matrix inversion operation in (18). This can be a task of a prohibitively high complexity when $M$ and $K$ are large as envisioned in large-scale MIMO systems. To address this issue, a TPE approach will be adopted in the next section.

\section{TPE-BASED PRECODER AND RECEIVER}

The common way to apply the TPE concept consists in replacing the matrix inverse by a weighted matrix polynomial with $J$ terms [6], [13]. Differently from the traditional approach, we propose in this work to apply the truncation artifice separately to each vector of the precoding and/or receiving matrix. The TPE technique in a per-UE basis was recently used in our work [17] to approximate the optimal linear precoding that minimizes the transmit power. Applying the TPE on a perUE basis, the precoding vector associated with UE $k$ writes as:

$$
\mathbf{g}_{k, \mathrm{TPE}}^{\mathrm{dl}}=\sqrt{\frac{p_{k, \mathrm{TPE}}}{K}} \frac{\mathbf{v}_{k, \mathrm{TPE}}}{\left\|\mathbf{v}_{k, \mathrm{TPE}}\right\|}
$$

with

$$
\mathbf{v}_{k, \mathrm{TPE}}=\sum_{\ell=0}^{J-1} w_{\ell, k}^{\mathrm{dl}}\left(\frac{\mathbf{H R H} \mathbf{H}^{H}}{K}\right)^{\ell} \mathbf{h}_{k}
$$

where $\frac{1}{K} p_{k, \mathrm{TPE}}$ is the power allocated to UE $k, \mathbf{R}_{k}=$ $\operatorname{diag}\left(\bar{q}_{1}, \ldots, \bar{q}_{K}\right)$. Plugging (19) into (3) and denoting by $\mathbf{w}_{k, \mathrm{dl}}=\left[w_{0, k}^{\mathrm{dl}}, \ldots, w_{J-1, k}^{\mathrm{dl}}\right]^{T} \in \mathbb{C}^{J \times 1}$, the SINR of UE $k$ can be rewritten as:

$$
\operatorname{SINR}_{k, \mathrm{TPE}}^{\mathrm{dl}}=\frac{\rho p_{k, \mathrm{TPE}} \frac{\mathbf{w}_{k, \mathrm{dl}}^{H} \mathbf{a}_{k} \mathbf{a}_{k}^{H} \mathbf{w}_{k, \mathrm{dl}}}{\mathbf{w}_{k, \mathrm{dl}}^{H} \mathbf{E}_{k} \mathbf{w}_{k, \mathrm{dl}}}}{\frac{\rho}{K} \sum_{i \neq k} p_{i, \mathrm{TPE}} \frac{\mathbf{w}_{i, \mathrm{dl}}^{H} \mathbf{B}_{k, i} \mathbf{w}_{i, \mathrm{dl}}}{\mathbf{w}_{i, \mathrm{dl}}^{H} \mathbf{E}_{i} \mathbf{w}_{i, \mathrm{dl}}}+1}
$$

where $\mathbf{a}_{k} \in \mathbb{C}^{J \times 1}, \mathbf{b}_{k} \in \mathbb{C}^{J \times 1}$, and $\mathbf{B}_{i, k} \in \mathbb{C}^{J \times J}$ are computed as:

$$
\begin{aligned}
{\left[\mathbf{a}_{k}\right]_{\ell} } & =\frac{1}{K} \mathbf{h}_{k}^{H}\left(\frac{\mathbf{H R H}^{H}}{K}\right)^{\ell} \mathbf{h}_{k} \\
{\left[\mathbf{B}_{k, i}\right]_{\ell, m} } & =\frac{1}{K} \mathbf{h}_{k}^{H}\left(\frac{\mathbf{H R H}}{K}\right)^{H} \mathbf{h}_{i} \mathbf{h}_{i}^{H}\left(\frac{\mathbf{H R H}}{K}\right)^{m} \mathbf{h}_{k}
\end{aligned}
$$

whereas the entries of $\mathbf{E}_{k} \in \mathbb{C}^{J \times J}$ are given by

$$
\left[\mathbf{E}_{k}\right]_{\ell, m}=\frac{1}{K} \mathbf{h}_{k}\left(\frac{\mathbf{H R H}}{K}\right)^{\ell+m} \mathbf{h}_{k} .
$$

The TPE concept is now applied to OLR. To this end, let $\left\{\frac{q_{k, \mathrm{TPE}}}{K}\right\}$ be the UL transmit powers. The receive vector associated to $\mathrm{UE} k$ is thus given by:

$$
\mathbf{g}_{k, \mathrm{TPE}}^{\mathrm{ul}}=\sum_{\ell=0}^{J-1} w_{\ell, k}^{\mathrm{ul}}\left(\frac{\mathbf{H R H} \mathbf{H}^{H}}{K}\right) \frac{\mathbf{h}_{k}}{\sqrt{K}} .
$$

Plugging (25) into (11) yields:

$$
\operatorname{SINR}_{k, \mathrm{TPE}}^{\mathrm{ul}}=\frac{\rho q_{k, \mathrm{TPE}} \mathbf{w}_{k, \mathrm{ul}}^{H} \mathbf{a}_{k} \mathbf{a}_{k}^{H} \mathbf{w}_{k, \mathrm{ul}}}{\sum_{i \neq k} \frac{\rho}{K} q_{i, \mathrm{TPE}} \mathbf{w}_{k, \mathrm{ul}}^{H} \mathbf{B}_{i, k} \mathbf{w}_{k, \mathrm{ul}}+\mathbf{w}_{k, u l}^{H} \mathbf{E}_{k} \mathbf{w}_{k, \mathrm{ul}}}
$$

where $\mathbf{w}_{k, \mathrm{ul}}=\left[w_{0, k}^{\mathrm{ul}}, \cdots, w_{J-1, k}^{\mathrm{ul}}\right]^{T}$ and $\mathbf{a}_{k}, \mathbf{B}_{k, i}$ and $\mathbf{E}_{k}$ are given by (22), (23) and (24), respectively. As for OLP and OLR, results from random matrix theory can be used to compute deterministic equivalents $\overline{\mathbf{a}}_{k}, \overline{\mathbf{B}}_{k, i}$ and $\overline{\mathbf{E}}_{k}$ of $\mathbf{a}_{k}$, $\mathbf{B}_{k, i}$ and $\mathbf{E}_{k}$ in the asymptotic regime in which $M, K$ grow large with the same pace. The details are omitted for space limitations but can be found in [16].

\section{OPTIMAL DESIGN OF THE TPE BASED PRECODER AND RECEIVER}

In the sequel, we compute the optimal weighting vectors $\mathbf{w}_{k, \mathrm{dl}}$ and $\mathbf{w}_{k, \mathrm{ul}}$ and the optimal transmit powers, in both uplink and downlink, that maximizes the minimum SINRs over all UEs. To begin with, we let

$$
\mathbf{c}_{k, \mathrm{dl}}=\frac{\overline{\mathbf{E}}_{k}^{\frac{1}{2}} \mathbf{w}_{k, \mathrm{dl}}}{\left\|\overline{\mathbf{E}}_{k}^{\frac{1}{2}} \mathbf{w}_{k, \mathrm{dl}}\right\|} \quad \mathbf{c}_{k, \mathrm{ul}}=\frac{\overline{\mathbf{E}}_{k}^{\frac{1}{2}} \mathbf{w}_{k, \mathrm{ul}}}{\left\|\overline{\mathbf{E}}_{k}^{\frac{1}{2}} \mathbf{w}_{k, \mathrm{ul}}\right\|}
$$

such that the SINR expressions in (21) and (26) reduce to:

$$
\overline{\operatorname{SINR}}_{k, \operatorname{TPE}}^{\mathrm{dl}}=\frac{\rho p_{k, \operatorname{TPE}} \mathbf{c}_{k, \mathrm{dl}}^{H} \overline{\mathbf{E}}_{k}^{-\frac{1}{2}} \overline{\mathbf{a}}_{k} \overline{\mathbf{a}}_{k}^{H} \overline{\mathbf{E}}_{k}^{-\frac{1}{2}} \mathbf{c}_{k, \mathrm{dl}}}{\sum_{i \neq k} \frac{\rho}{K} p_{i, \operatorname{TPE}} \mathbf{c}_{i, \mathrm{dl}}^{H} \overline{\mathbf{E}}_{i}^{-\frac{1}{2}} \overline{\mathbf{B}}_{k, i} \overline{\mathbf{E}}_{i}^{-\frac{1}{2}} \mathbf{c}_{i, \mathrm{dl}}+1}
$$




$$
\begin{gathered}
q_{k, \mathrm{TPE}}^{\star}=\frac{\gamma_{k} K P_{\max }}{\sum_{\ell=1}^{K} \frac{\gamma_{\ell} \overline{\mathbf{a}}_{k}^{T} \overline{\mathbf{E}}_{k}^{-\frac{1}{2}}\left(\sum_{i \neq k} \frac{\rho}{K} q_{i, \mathrm{TPE}}^{\star} \overline{\mathbf{E}}_{k}^{-\frac{1}{2}} \overline{\mathbf{B}}_{i, k} \overline{\mathbf{E}}_{k}^{-\frac{1}{2}}+\mathbf{I}_{J}\right)^{-1} \overline{\mathbf{E}}_{k}^{-\frac{1}{2}} \overline{\mathbf{a}}_{k}}{\overline{\mathbf{E}}_{\ell}^{-\frac{1}{2}}\left(\sum_{j \neq \ell} \frac{\rho}{K} q_{j, \mathrm{TPE}}^{\star} \overline{\mathbf{E}}_{\ell}^{-\frac{1}{2}} \overline{\mathbf{B}}_{j, \ell} \overline{\mathbf{E}}_{\ell}^{-\frac{1}{2}}+\mathbf{I}_{J}\right)^{-1} \overline{\mathbf{E}}_{\ell}^{-\frac{1}{2}} \tilde{\mathbf{a}}_{\ell}}} \\
\mathbf{c}_{k, \mathrm{ul}}^{\star}=\mathbf{c}_{k, \mathrm{dl}}^{\star}=\frac{\left(\sum_{i \neq k} \frac{\rho}{K} q_{i, \mathrm{TPE}}^{\star} \overline{\mathbf{E}}_{k}^{-\frac{1}{2}} \overline{\mathbf{B}}_{i, k} \overline{\mathbf{E}}_{k}^{-\frac{1}{2}}+\mathbf{I}_{J}\right)^{-1} \overline{\mathbf{E}}_{k}^{-\frac{1}{2}} \overline{\mathbf{a}}_{k}}{\left\|\left(\sum_{i \neq k} \frac{\rho}{K} q_{i, \mathrm{TPE}}^{\star} \overline{\mathbf{E}}_{k}^{-\frac{1}{2}} \overline{\mathbf{B}}_{i, k} \overline{\mathbf{E}}_{k}^{-\frac{1}{2}}+\mathbf{I}_{J}\right)^{-1} \overline{\mathbf{E}}_{k}^{-\frac{1}{2}} \overline{\mathbf{a}}_{k}\right\|}
\end{gathered}
$$

and

$$
\overline{\operatorname{SINR}}_{k, \mathrm{TPE}}^{\mathrm{ul}}=\frac{\rho q_{k, \mathrm{TPE}} \mathbf{c}_{k, \mathrm{ul}}^{H} \overline{\mathbf{E}}_{k}^{-\frac{1}{2}} \overline{\mathbf{a}}_{k} \overline{\mathbf{a}}_{k}^{H} \overline{\mathbf{E}}_{k}^{-\frac{1}{2}} \mathbf{c}_{k, \mathrm{ul}}}{\sum_{i \neq k} \frac{\rho}{K} q_{i, \mathrm{TPE}} \mathbf{c}_{k, \mathrm{ul}}^{H} \overline{\mathbf{E}}_{k}^{-\frac{1}{2}} \overline{\mathbf{B}}_{i, k} \overline{\mathbf{E}}_{k}^{-\frac{1}{2}} \mathbf{c}_{k, \mathrm{ul}}+1}
$$

after replacing $\mathbf{a}_{k}, \mathbf{B}_{k, i}$ and $\mathbf{E}_{k}$ with $\overline{\mathbf{a}}_{k}, \overline{\mathbf{B}}_{k, i}$ and $\overline{\mathbf{E}}_{k}$. The parameters $\left\{\mathbf{c}_{k, \mathrm{dl}}\right\},\left\{\mathbf{c}_{k, \mathrm{ul}}\right\},\left\{p_{k, \mathrm{TPE}}\right\}$ and $\left\{q_{k, \mathrm{TPE}}\right\}$ are computed as the solution of the following optimization problems:

$$
\max _{\left\{\mathbf{c}_{k, \mathrm{~d} l}\right\},\left\{p_{k, \mathrm{TPE}}\right\}} \min _{k} \frac{\overline{\mathrm{SINR}}_{k, \mathrm{TPE}}^{\mathrm{dl}}}{\gamma_{k}} \text { s.t } \frac{1}{K} \sum_{k=1}^{K} p_{k, \mathrm{TPE}}=P_{\max }
$$

and

$$
\max _{\left\{\mathbf{c}_{k, \mathrm{ul}}\right\},\left\{q_{k, \mathrm{TPE}}\right\}} \min _{k} \frac{\overline{\mathrm{SINR}}_{k, \mathrm{TPE}}^{\mathrm{ul}}}{\gamma_{k}} \text { s.t. } \frac{1}{K} \sum_{k=1}^{K} q_{k, \mathrm{TPE}}=P_{\max }
$$

which have the same structure of (5) and (10). Following similar arguments, it turns out that the solution is such that all the weighted asymptotic SINRs are equal to the same value $\tau_{\mathrm{TPE}}^{\star}$ :

$$
\tau_{\mathrm{TPE}}^{\star}=\frac{\overline{\operatorname{SINR}}_{k, \mathrm{TPE}}^{\mathrm{ul}}}{\gamma_{k}}=\frac{\overline{\operatorname{SINR}}_{k, \mathrm{TPE}}^{\mathrm{dl}}}{\gamma_{k}} \quad \forall k .
$$

The optimal values $q_{k, \mathrm{TPE}}^{\star}$ are obtained as the unique solution of the set of equations given by (27) whereas the weighting vectors take the form in (28). The computation of $q_{k, \mathrm{TPE}}^{\star}$ requires matrix inversions whose complexity depends on $J$. However, $J$ is small and does not need to scale with the values of $M$ and $K$. Thus, the computation of $q_{k, \text { TPE }}^{\star}$ is not very computationally demanding. The optimal power vector $\mathbf{p}_{\mathrm{TPE}}^{\star}$ is such that the weighted SINRs in the uplink are all equal to $\tau_{\mathrm{TPE}}^{\star}$. This yields:

$$
\mathbf{p}_{\mathrm{TPE}}^{\star}=\frac{\tau_{\mathrm{TPE}}^{\star}}{\rho}\left(\mathbf{I}_{K}-\tau_{\mathrm{TPE}}^{\star} \boldsymbol{\Gamma}_{\mathrm{TPE}} \mathbf{F}_{\mathrm{TPE}}\right)^{-1} \boldsymbol{\Gamma}_{\mathrm{TPE}} \mathbf{1}_{K}
$$

where

$$
\boldsymbol{\Gamma}_{\mathrm{TPE}}=\operatorname{diag}\left\{\left(\mathbf{c}_{k, \mathrm{dl}}^{H} \overline{\mathbf{E}}_{k}^{-\frac{1}{2}} \overline{\mathbf{a}}_{k} \overline{\mathbf{a}}_{k}^{H} \overline{\mathbf{E}}_{k}^{-\frac{1}{2}} \mathbf{c}_{k, \mathrm{dl}}\right)^{-1}\right\}_{k=1}^{K}
$$

and

$$
\left[\mathbf{F}_{\mathrm{TPE}}\right]_{i, k}= \begin{cases}0 & \text { if } k=i \\ \frac{1}{K} \mathbf{c}_{i, \mathrm{dl}}^{H} \overline{\mathbf{E}}_{k}^{-\frac{1}{2}} \overline{\mathbf{B}}_{k, i} \overline{\mathbf{E}}_{k}^{-\frac{1}{2}} \mathbf{c}_{i, \mathrm{dl}} & \text { if } k \neq i .\end{cases}
$$

From the above results, it follows that the TPE-based schemes have the same structure as OLP and OLR. However, the former have the following main advantages: $(i)$ the optimal parameters depend only on the large scale channel statistics and can be thus computed beforehand, or at least be updated at the rate of the change of the channel statistics; (ii) they allow a considerable complexity reduction since they do not require the computation of a matrix inverse. This results into only about $\mathcal{O}(K M)$ arithmetic operations to be compared with the $\mathcal{O}\left(K^{2} M\right)$ operations required by OLP and OLR. For more details about complexity analysis, we refer the reader to [13] where the complexity of the RZF precoding is computed and compared with the complexity of the TPE precoding.

\section{Simulation Results}

Numerical results are now used to compare OLP and OLR with the corresponding TPE-based schemes and to validate the asymptotic analysis. The UEs are assumed to be uniformly distributed in a cell with radius $250 \mathrm{~m}$. The path loss $\beta_{k}$ is modelled as $\beta_{k}=\left(1+\left(d_{k} / d_{0}\right)^{\delta}\right)^{-1}$ where $d_{k}$ denotes the distance of UE $k$ from the BS whereas $\delta=3.8$ and $d_{0}=30 \mathrm{~m}$. The analysis is conducted in terms of the average achievable rate per UE given by:

$$
r=\frac{1}{K} \sum_{k=1}^{K} \mathbb{E}\left[\log _{2}\left(1+\operatorname{SINR}_{k}\right)\right]
$$

where the expectation is taken with respect to the different channel realization. We set $\rho=20 \mathrm{~dB}$ and assume that the UEs priorities $\left\{\gamma_{k}\right\}$ are randomly chosen from the interval $[1,2]$. Markers are used to represent the asymptotic results whereas the error bars indicate the standard deviation of the Monte Carlo results.

Fig. 1 reports the downlink average rate per UE as a function of the power constraint $P_{\max }$ when $K=32$ and $M=128$. Note that when $J=1$, the US-TPE precoder coincide with the maximum ratio transmission (MRT) precoding. The results show that $J=2$ is sufficient to achieve the same performance of OLP when $P_{\max }$ is relatively small. Larger values are needed when $P_{\max }$ increases. This is due to the fact that when $P_{\max }$ gets higher, the SINR of the OLP increases. A higher number of degrees of freedom is thus required in order to approximate the performance of the OLP.

Fig. 2 illustrates the uplink average rate per UE vs. $M$ for different values of the power constraint $P_{\max }$. As seen, with $J=2$ the TPE-based receiver provides interesting 


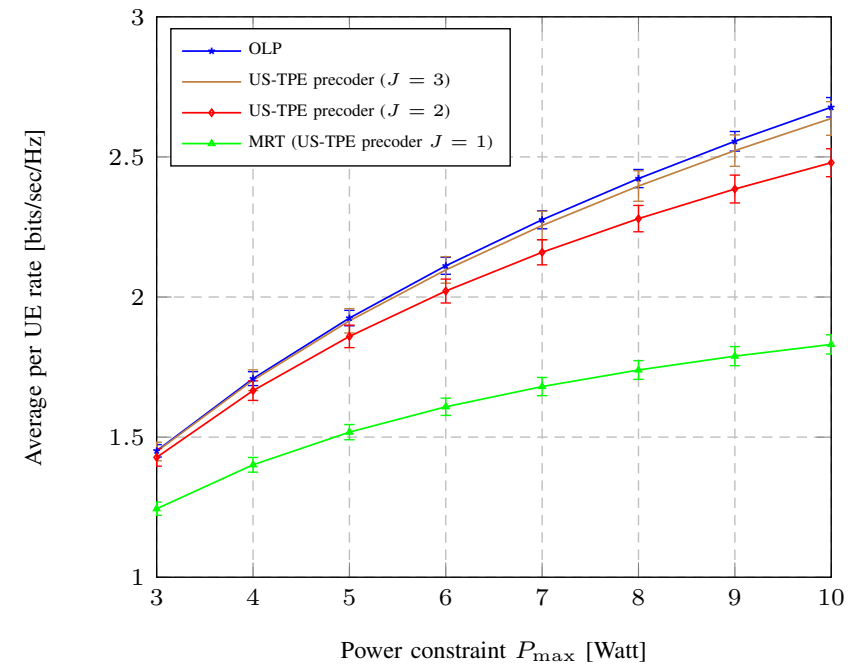

Fig. 1. Average per UE rate vs. power constraint $P_{\max }$ when $K=32$, $M=128$ and $\rho=20 \mathrm{~dB}$.

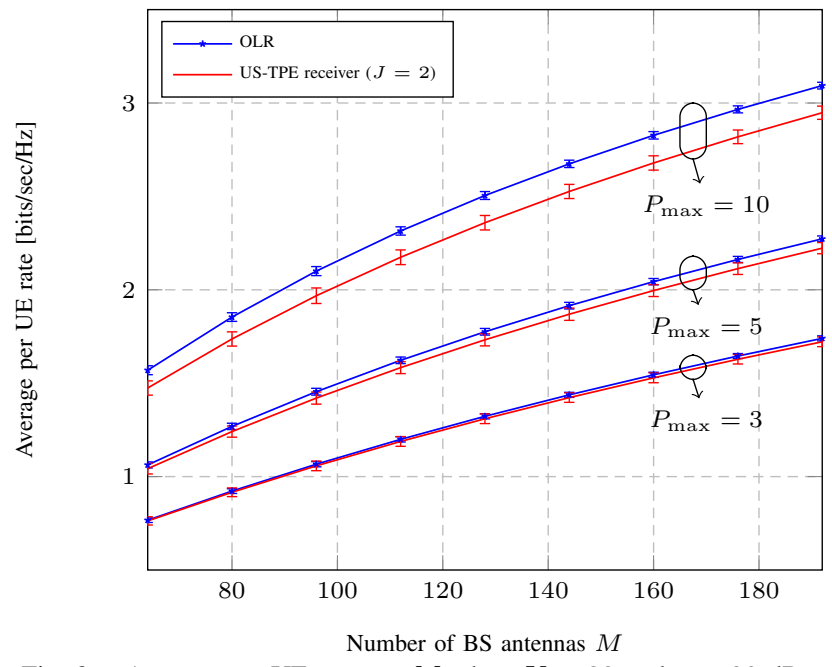

Fig. 2. Average per UE rate vs. $M$ when $K=32$ and $\rho=20 \mathrm{~dB}$.

performance compared OLR for all the values of $M$. Besides, as in downlink, the gap is getting larger when $P_{\max }$ is getting higher. However, we can increase the truncation order $J$ to enhance the performance of the proposed receiver and get closer to the OLR. Also, it is important to notice from this figure that the gap between the proposed receiver and the OLR becomes constant for large values of $M$.

\section{CONCLUSION}

In this work, we focused on the design of the linear TPE precoder and receiver in order to achieve the same performance as the optimal linear precoder (OLP) and the optimal linear receiver (OLR) that maximizes the minimum SINR. Differently from the traditional approach, the TPE concept is applied on a per-UE basis. In order to facilitate the design of the TPE transceivers, we considered the asymptotic regime in which the number of antennas at the BS and that of users grow simultaneously large with the same pace. The use of this assumption allowed us to approximate the
SINRs by deterministic quantities that depend only on the channel statistics. The TPE weights are then optimized in order to maximize the asymptotic minimum SINRs over all users. Numerical results are presented in order to illustrate the efficiency of TPE based schemes in achieving close-to performances to the optimal schemes.

\section{REFERENCES}

[1] T.L. Marzetta, "Noncooperative cellular wireless with unlimited numbers of base station antennas," IEEE Trans. Commun., vol. 9, no. 11, pp. 3590-3600, Nov. 2010.

[2] J. Hoydis, S. ten Brink, and M. Debbah, "Massive MIMO in the UL/DL of cellular networks: How many antennas do we need?," IEEE J. Sel. Areas Commun., vol. 31, no. 2, pp. 160-171, Feb. 2013.

[3] F. Rusek, D. Persson, B.K. Lau, E.G. Larsson, T.L. Marzetta, O. Edfors, and F. Tufvesson, "Scaling up MIMO: Opportunities and challenges with very large arrays," IEEE Signal Process. Mag., vol. 30, no. 1, pp. 40-60, Jan. 2013.

[4] E. G. Larsson, O. Edfors, F. Tufvesson, and T. L. Marzetta, "Massive mimo for next generation wireless systems," IEEE Commun. Mag., vol. 52, no. 2, pp. 186-195, Feb. 2014

[5] E. Björnson, G. Zheng, M. Bengtsson, and B. Ottersten, "Robust monotonic optimization framework for multicell miso systems," IEEE Trans. Signal Process., vol. 60, no. 5, pp. 2508-2523, Jun. 2012.

[6] H. Sifaou, A. Kammoun, L. Sanguinetti, M. Debbah, and M-S. Alouini, "Power efficient low complexity precoding for massive MIMO systems," in GlobalSIP 2014, Atalanta, Georgia, 2014.

[7] A. Wiesel, Y. Eldar, and S. Shamai, "Linear precoding via conic optimization for fixed mimo receivers," IEEE Transactions on Signal Processing, vol. 54, pp. 161-176, 206.

[8] L. Sanguinetti, A.L. Moustakas, E. Bjornson, and M. Debbah, "Large system analysis of the energy consumption distribution in multi-user MIMO systems with mobility," IEEE Trans. Wireless Commun., vol. 14, no. 3, pp. 1730 - 1745, March 2015.

[9] D. W. H. Cai, T. Q. S. Quekand, C. Wei Tan, and S. H. Low, "Maxmin sinr coordinated multipoint downlink transmission - duality and algorithms," IEEE Transactions on Signal Processing, vol. 60, pp. 53845395, 2012.

[10] Y. Huang, C. Wei Tan, and B. D. Rao, "Joint beamforming and power control in coordinated multicell: Max-min duality, effective network and large system transition," IEEE Transactions on Wireless Communications, pp. 2730-2742, 2013.

[11] R. Zakhour and S.V. Hanly, "Base station cooperation on the downlink: Large system analysis," IEEE Trans. Inf. Theory, vol. 58, no. 4, pp. 2079-2106, Apr. 2012.

[12] L. Sanguinetti, R. Couillet, and M. Debbah, "Large system analysis of base station cooperation for power minimization," IEEE Trans. Wireless Commun., Sept. 2015, submitted, [Online] http://arxiv.org/abs/1509.00731.

[13] A. Müller, A. Kammoun, E. Björnson, and M. Debbah, "Linear precoding based on truncated polynomial expansion: Reducing complexity in massive MIMO," IEEE Trans. Inf. Theory, July 2014, Submitted, arXiv: 1310.1806

[14] G. Sessler and F. Jondral, "Low complexity polynomial expansion multiuser detector for CDMA systems," IEEE Trans. Veh. Technol., vol. 54, no. 4, pp. 1379-1391, July 2005.

[15] N. Shariati, E. Björnson, M. Bengtsson, and M. Debbah, "Lowcomplexity channel estimation in large-scale MIMO using polynomial expansion," in Proc. IEEE Int. Symp. Personal, Indoor and Mobile Radio Commun. (PIMRC), 2013.

[16] H. Sifaou, A. Kammoun, L. Sanguinetti, M. Debbah, and M-S. Alouini, "Low complexity precoding and receiver for the max-min sinr in multiuser massive MIMO systems," Under preparation, 2016.

[17] H. Sifaou, A. Kammoun, L. Sanguinetti, M. Debbah, and M-S. Alouini, "Polynomial expansion of the power minimization precoder in largescale MIMO systems," in ICC 2016, Kuala Lumpur, Malaysia, 2016. 